\title{
A 3D PARALLEL BEAM DYNAMICS CODE FOR MODELING HIGH BRIGHTNESS BEAMS IN PHOTOINJECTORS*
}

\author{
J. Qiang ${ }^{\dagger}$, S. Lidia, R. Ryne, LBNL, Berkeley, CA 94547, USA \\ C. Limborg, SLAC, Menlo Park, CA 94025, USA
}

\begin{abstract}
In this paper we report on IMPACT-T, a 3D beam dynamics code for modeling high brightness beams in photoinjectors and rf linacs. IMPACT-T is one of the few codes used in the photoinjector community that has a parallel implementation, making it very useful for high statistics simulations of beam halos and beam diagnostics. It has a comprehensive set of beamline elements, and furthermore allows arbitrary overlap of their fields. It is unique in its use of space-charge solvers based on an integrated Green function to efficiently and accurately treat beams with large aspect ratio, and a shifted Green function to efficiently treat image charge effects of a cathode. It is also unique in its inclusion of energy binning in the space-charge calculation to model beams with large energy spread. Together, all these features make IMPACT-T a powerful and versatile tool for modeling beams in photoinjectors and other systems. In this paper we describe the code features and present results of IMPACT-T simulations of the LCLS photoinjectors. We also include a comparison of IMPACT-T and PARMELA results.
\end{abstract}

\section{INTRODUCTION}

High brightness, low emittance electron beams from photoinjectors have important applications in next generation light sources and international linear collider. Simplified theoretical models have been used to provide some guidelines for photoinjector design $[1,2]$. For a systematic machine design and optimization, it still heavily depends on the use of self-consistent computer simulations. A number of computer codes have been developed in the past years to simulate beam transport inside photoinjectors $[3,4,5,6,7,8,9]$. As far as we know, a code which can efficiently and accurately handle a three-dimensional beam with large-aspect ratio and large energy spread including possible image-charge effects is still not available on parallel computer. In this paper, we report on developing a threedimensional quasi-static particle-in-cell code to simulate the beam transport through the photoinjector using a shifted integrated Green function method for space-charge calculation. Using a shifted-integrated Green function, we can accurately and efficiently calculate the three-dimensional space-charge forces using a fast Fouier transform (FFT)

* Work supported by a Scientifi c Discovery through Advanced Computing project, "Advanced Computing for 21st Century Accelerator Science and Technology," which is supported by the US DOE/SC Offi ce of High Energy Physics and the Offi ce of Advanced Scientifi c Computing Research.

† jqiang@lbl.gov method. The large energy spread is handled by dividing the beam into multiple energy bins and the space-charge forces are calculated for each bin and are summed together before being interpolated to individual particle. Besides the efficient numerical algorithm for calculation the 3D spacecharge forces, we have also implemented this model on parallel computers. This enables us to run a simulation with both high resolution and fast return time.

\section{PHYSICAL MODEL AND COMPUTATIONAL METHODS}

The physical model assumed in the code is a quasistatic charged particle beam subject to the external acceleration and focusing and the space-charge forces from the beam itself. Radiation effects of the beam are not included. Comparison studies with and without the radiation effects suggest that the radiation effects should not be significant inside photoinjectors [10,11]. The simulation starts from generating a three-dimensional beam bunch behind the photocathode according to the laser pulse length and distribution. This bunch is then moved out of the cathode within the given emission time. During the process of emission, the space-charge forces of particles outside the cathode are included together with external fields from RF cavity and solenoid magnet. Besides the RF/DC cavity and solenoid magnet beam line elements, the IMPACT-T code also has a dipole magnet, a quadrupole magnet, a multipole magnet (sextupole, octupole, and decapole) element. The external fields can be supplied in the form of discrete data on a three-dimensional Cartesian mesh or cylindrical coordinate mesh. They can also be supplied in the form of Fourier expansion coefficients on the axis when the fields have azimuthal symmetry. These elements can be arranged with arbitrary longitudinal overlap so that a traveling wave structure (including both end cells) can be modeled using four overlapped standing wave structures [12].

Macroparticles inside the photoinjector are advanced self-consistently using a particle-in-cell approach. Here, following a second-order leap-frog algorithm, the particles are drifted half time step; the particles are collected and deposited onto a three-dimensional grid; the Poisson equation is solved in the beam frame; the electric and magnetic fields are obtained in the lab frame through the Lorentz transformation; the particle momenta are updated using both the space-charge fields and external fields for one time step according to the Lorentz equation; the particles are drifted another half time step. This procedure is repeated for many time steps until the beam is out of the computational domain. 
To calculate the space-charge forces, we need to solve the three-dimensional Poisson equation. The solution of Poisson's equation can be written as:

$$
\left.\phi(x, y, z)=\int G(x, \bar{x}, y, \bar{y}, z, \bar{z}) \rho(\bar{x}, \bar{y}, \bar{z}) d \bar{x} d \bar{y} d \bar{k} 1\right)
$$

where $G$ is Green's function, $\rho$ is the charge density distribution function. For the case of open boundary conditions, the Green function is given by:

$$
\left.G(x, \bar{x}, y, \bar{y}, z, \bar{z})=\frac{1}{\sqrt{(x-\bar{x})^{2}+(y-\bar{y})^{2}+(z-\bar{z})^{2}}}\right)
$$

The computational cost of above convolution by a direct numerical summation can be very expensive and scales as $N^{6}$, where $N$ is number of grid points in each dimension. Fortunately, this convolution can be replaced by a cyclic convolution in a double-gridded computational domain. The resultant new convolution can be computed very efficiently using an FFT method and the computational cost scales as $N^{3} \log (N)$ [13].

In above algorithm, both the Green function and the charge density distribution are discretized on the grid. For a beam with low aspect ratio, this algorithm works well. However, during the emission of electrons out of the cathode, the beam can have a very large transverse to longitudinal ratio. Under this situation, the direct use of the Green function at each mesh point is not efficient since it requires a large number of mesh points along the transverse direction in order to get sufficient resolution for the Green function along that direction. However, if we assume that the charge density function is uniform within each cell centered at the grid point $\left(x_{i}, y_{j}, z_{k}\right)$, we can define an effective Green function as

$$
\begin{array}{r}
\bar{G}_{\left(x_{i}-x_{i^{\prime}}, y_{j}-y_{j^{\prime}}, z_{k}-z_{k^{\prime}}\right)}= \\
\int d x^{\prime} \int d y^{\prime} \int d z^{\prime} G\left(x_{i}-x^{\prime}, y_{j}-y^{\prime}, z_{k}-z^{\prime}\right)
\end{array}
$$

This integral can be calculated analytically in a closed form [14].

For a conducting photo-cathode, the image charge of a beam near the cathode is not negligible. The space-charge effects from the image charge and from the beam should be included. To find the space-charge forces on the beam from the image charge by the standard Green function method, we need to solve the Poisson equation with a computational domain containing both the image charge and the beam. This can be very inefficient since only the fields within the beam are needed and the fields outside of the beam are not used. This inefficiency can be avoided by using a shiftedGreen function [15]. Using the shifted Green function, the center of the field domain is shifted to the center of the particle domain. The range of $x, y$ and $z$ cover both the particle domain and the field domain in one computational domain. The FFT is used to calculate the cyclic convolution in the previous algorithm can be used to calculate the potential in the shifted field domain. To test this solver, we have calculated the potential distribution on the axis from the image charge of a round beam. The numerical results are given in Fig. 1 together with the analytical solution. It is seen that the numerical solution and the analytical solution agree with each other very well.

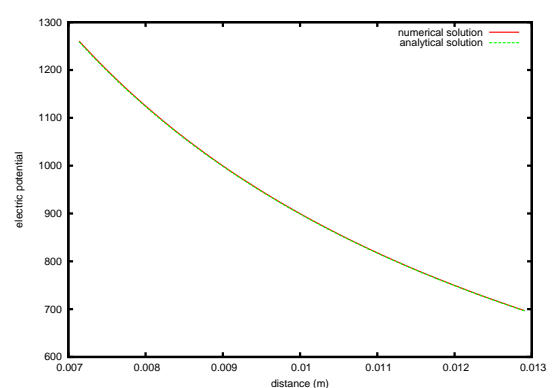

Figure 1: Image charge potential on the axis for a round beam from the shifted Green function solution together with the analytical solution.

\section{APPLICATIONS}

Before the application to the LCLS photoinjector study, we have done a benchmark between the IMPACT-T and the PARMELA using an S band photoinjector at SLAC [16]. The evolution of transverse and longitudinal rms size is given in Figs. 2 and 3. The evolution of normalized rms emittance and relative rms energy deviation is given in Figs. 4 and 5.

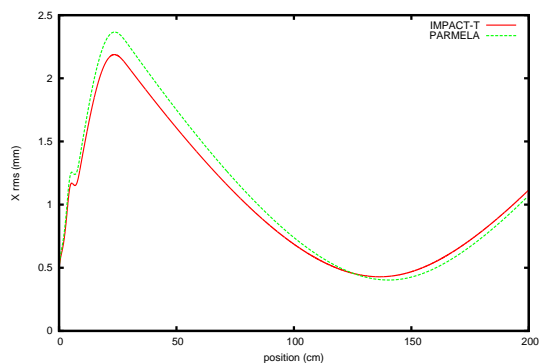

Figure 2: Transverse rms size as a function of distance.

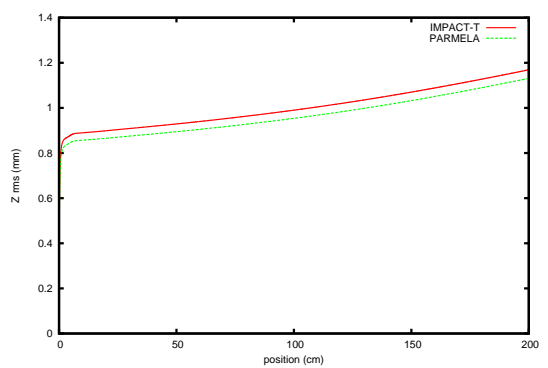

Figure 3: Longitudinal rms size as a function of distance.

We see that the simulation results from both codes agree with each other pretty well. The differences observed here could be due to the different way of treating the electron 


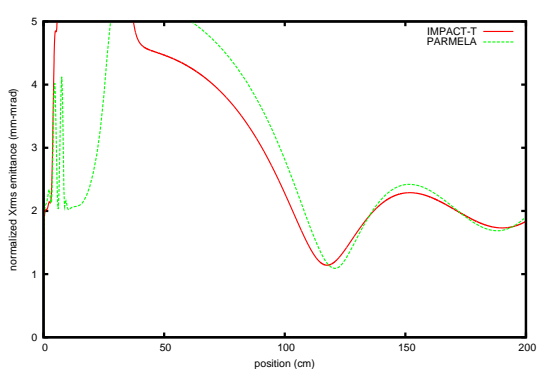

Figure 4: Transverse normalized rms emittance as a function of distance.

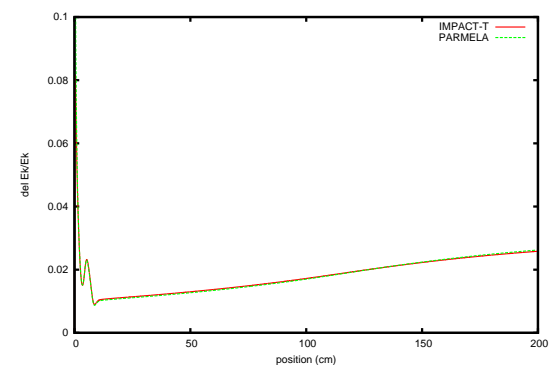

Figure 5: Longitudinal rms size as a function of distance.

emission, the exact RF driven phase used, and the numerical method to calculate the space-charge forces.

As an application, we have used the IMPACT-T code to simulate the beam transport through the designed LCLS photoinjector. The simulation was done using one hundred thousand macroparticles with $64 \times 64 \times 64$ mesh points. The photoinjector consists of a one-and-half cell $2.856 \mathrm{GHz}$ $\mathrm{S}$ band gun, a 85 cell traveling wave structure, and two solenoid magnets for initial emittance compensation at low energy and transverse focusing. The electrons out of the photocathode is accelerated to $62 \mathrm{MeV}$ after the traveling wave structure. To emulate a real operation, we have assumed that the initial beam out of the cathode has a $100 \mathrm{um}$ horizontal offset. This centroid offset is corrected by steering magnets so that at the entrance to traveling wave structure, the bunch centroid is set back to zero. Fig. 6 shows the normalized rms emittance as a function of distance from the simulation. Compared with the case without initial off-

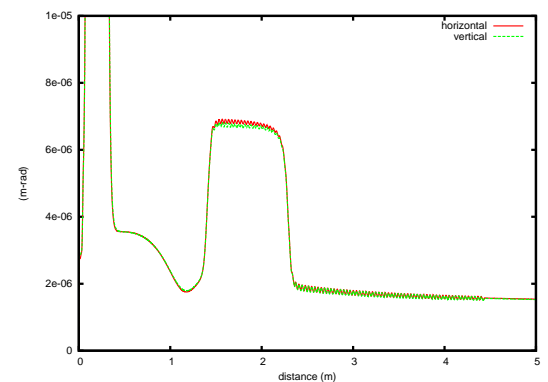

Figure 6: Transverse normalized rms emittance as a function of distance. set, the transverse emittance is not significantly affected by the initial 100 um offset. The final rms emittance is below 2 $\mathrm{mm}$-mrad. The energy spread of the beam could affect the beam stability and lasing in the LCLS. Fig. 7 gives the final longitudinal phase space distribution in $Z-P_{z}$ plane. The

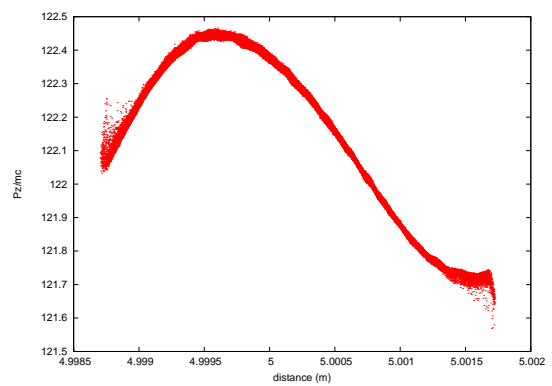

Figure 7: Longitudinal phase space distribution after the first traveling wave linac.

total correlated rms energy spread at the output $62 \mathrm{MeV}$ is about $150 \mathrm{KeV}$, while the uncorrelated rms energy spread varies from a minimum $3 \mathrm{KeV}$ near the center to $17 \mathrm{KeV}$ at the tail of the beam.

\section{REFERENCES}

[1] K. J. Kim, Nucl. Instr. and Meth. A 275 (1989) 201.

[2] L. Serafi ni and J. B. Rosenzweig, Phys. Rev. E 55, (1997) 7565.

[3] L. Young, J. Billen, PARMELA, LANL Codes, laacgl.lanl.gov/laacg/services/parmela.html.

[4] K. Flottmann and P. Piot, Proceedings of EPAC 2002 (Paris), 1798-1800 (2002).

[5] S. B. Van der Geer, M. J. de Loos, Proc. of the 1997 Part. Acc. Conf., Vancouver, Canada, IEEE (1998) p. 2577.

[6] L. Giannessi et al., AIP CP 413 (1997) 301.

[7] J. L. Coacolo et al., Nucl. Instr. and Meth. A 393 (1997) 430.

[8] L. Serafi ni and C. Pagani, Proc. of IEPAC, Rome, 1998, p. 866.

[9] Y.Batygin, ISSN 1344-3877, RIKEN-AF-AC-17 (2000), 81 p.

[10] L. Serafi ni, in The Physics of High Brightness Beams, ed. by J. Rosenzweig and L. Serafi ni, World Scientifi c (2000), p. 27.

[11] E. Colby, V. Ivanov, Z. Li, C. Limborg, Proc. of the 7th ICAP, Michigan, USA, 15-18 October, 2002, p. 47.

[12] G. A. Loew, R. H. Miller, R. A. Early and K. L. Bane, SLAC-PUB-2295 (1979).

[13] R. W. Hockney and J. W. Eastwood, Computer Simulation Using Particles, Adam Hilger: New York, 1988.

[14] R. D. Ryne, in ICFA Beam Dynamics Mini Workshop on Space Chrage Simulation, Trinity College, Oxford, 2-4 April 2003.

[15] J. Qiang, M. A. Furman, R. D. Ryne, Phys. Rev. Special Topics - Accel. Beams, 5 (2002) 104402.

[16] C. Limborg, Y. Batygin, et al., Proceedings of PAC 2003 (Portland), 2003, p. 3548. 